\title{
Managing change in general practice: a step by step guide
}

\author{
Nicki Spiegal, Elizabeth Murphy, Ann-Louise Kinmonth, Fran Ross, John Bain, Robin Coates
}

Over the past five years general practitioners have been challenged by proposals from both the government and the Royal College of General Practitioners to fundamentally change the administrative structure and day to day running of general practice. ${ }^{1-5}$ Changes range from individual practice based innovations, such as developing structured surveillance for chronic disorders and improving the provision of preventive services, to broader initiatives, such as performance review and an emphasis on medical audit.

Though much attention has been paid to the content of such change, less energy has been directed towards establishing how change can be managed most effectively. Experience in industry suggests that even the most rational change can be obstructed if its implementation is either badly planned or inadequately negotiated. Several models have been developed to assist the successful management of change,$^{67}$ some of which have been applied to various aspects of the NHS. ${ }^{8-10}$ We discuss how one such model, which was first developed in industry, has been usefully applied in general practice settings.

We worked with a management consultant (RC), who has considerable industrial experience, to develop and adapt a model for managing change for general practice; the model emphasises a team approach. We have applied this model to different situations in general practice, including introducing screening services and surveillance of chronic disorders, incorporating new technology into practice administration, and improving liaison and effective working relationships between different professional groups. ${ }^{112}$ The model can be used either by a practice that already functions as a cohesive team or by a person within the practice who wishes to move towards a team based decision in introducing change.

In practices that already function well as a team, with regular meetings of the whole team in which each member's contribution is recognised and respected, the process of shared decision making about an innovation can be started with few of the preliminary steps described here. However, more often an innovation is sponsored by a single person. In this case the strategies described can be used to introduce an idea to other team members, to negotiate agreement and implementation, if appropriate, in such a way that it strengthens the team and has maximum chance of success.

\section{Getting started}

The first step in our model of introducing change is to obtain relevant information from various sources. We will consider the key questions separately.

Has it been tried before?-Both literature searches and personal contact with those who have experience of similar initiatives can provide useful information to share with the practice team and reassure members about the value of the proposed innovation. Important insights can be obtained whether or not such attempts at innovation have been successful.

Is the practice ready for change? - Research in industry suggests that innovations are more successful in organisations where there are "slack resources." 12 In most general practices time, money, staff, and space are stretched to the limit and innovation may be possible only if new resources can be found or existing ones reallocated. For example, if available nurse time is already fully committed a nurse coordinated diabetes clinic cannot be introduced without generating more nurse sessions or restructuring the nurse's existing commitments. Innovations fail when enthusiasm ignores such practical constraints.

Who makes the decisions? ${ }^{13}$-If a senior partner makes all the decisions change may be introduced without overt opposition, but this can result in failure in the longer term as without adequate consultation other members of the team may be less committed to the change. Shared decision making, although sometimes

\section{Box 1: Techniques for encouraging collaboration in innovation}

1 Be explicit about why you think it is important to involve the team member

2 Behave with trust towards your colleagues, expecting them to be able to look at the idea objectively and not ridicule it

3 Be receptive to both positive and negative responses to your ideas and be seen to value each person's opinion even if it contradicts your original thinking. Agreement achieved after negative reactions have been identified and explored is more powerful than premature agreement obtained by evading potential conflict

4 When potential benefits are identified it is important that these should be seen as realistic and obtainable

5 When team members identify the potential costs to themselves these must be addressed, however trivial they may seem. Strategies for minimising such costs can then be explored. Team members will often need this opportunity to express and deal with the fear which may accompany proposals for change

more difficult and time consuming, has greater potential for success. In both these cases, recognising how decisions are made in the practice is helpful. It is also important to consider the external influences which may affect the success of the innovation. These may include government policy, patient demand, the family health services authority, and consultant colleagues.

Who are the key people? - Any person or group who may be affected by a proposed change is likely to have the power to promote or obstruct it. Experience in industry suggests that even those with low status can wield power, particularly the power to obstruct change. ${ }^{13}$ For example, doctors could invest considerable time and effort in introducing a computer into their practice. If, however, reception staff have the task of operating the computer imposed on them they may be reluctant to cooperate. Effective negotiation depends on early identification and involvement of everyone who may be affected. This builds ownership and commitment to the proposed change and is the first step towards developing a "shared vision."

Who might support the change? - After identifying all those who have a "stake" in the innovation (stakeholders), it may help to meet them individually to share ideas and to discover how they respond to the proposed change. Given the pressure on time in general practice,
Correspondence to: Nicki Spiegal. 
individual meetings may seem unrealistic. However, we have found that allocating this time at an early stage proves economical in the long run. Innovations that are partly implemented and then fail through lack of adequate negotiation waste large amounts of time..$^{15}$ The challenge of such meetings is to set them up so that they are opportunities for constructive discussion that allow deeper understanding of each person's perspectives and result in an innovation which is better than anybody could have reached working alone. A useful

\section{Box 2: Creating a domainal map}

1 Draw six concentric circles

2 Divide these circles into as many segments as there are stakeholders

3 Enter details in each circle:

- Circle 1: Title of proposed change

- Circle 2: Names of stakeholders

- Circle 3: Current involvement of each stakeholder in situation where change is proposed. (This enables reflection on the agenda of each stakeholder)

- Circle 4: Future benefits of the change for each stakeholder. (Enables clarity about advantages of the change to each stakeholder)

- Circle 5: Potential costs to each stakeholder of the proposed change (such costs may include time, money, loss of status, etc)

- Circle 6: Unaware "wrecking power" of each stakeholder. (This is the potential power of stakeholders to obstruct the change. There is no assumption here that stakeholders are likely to use this power maliciously. Simply pursuing their own legitimate goals within the organisation may jeopardise your proposed change)

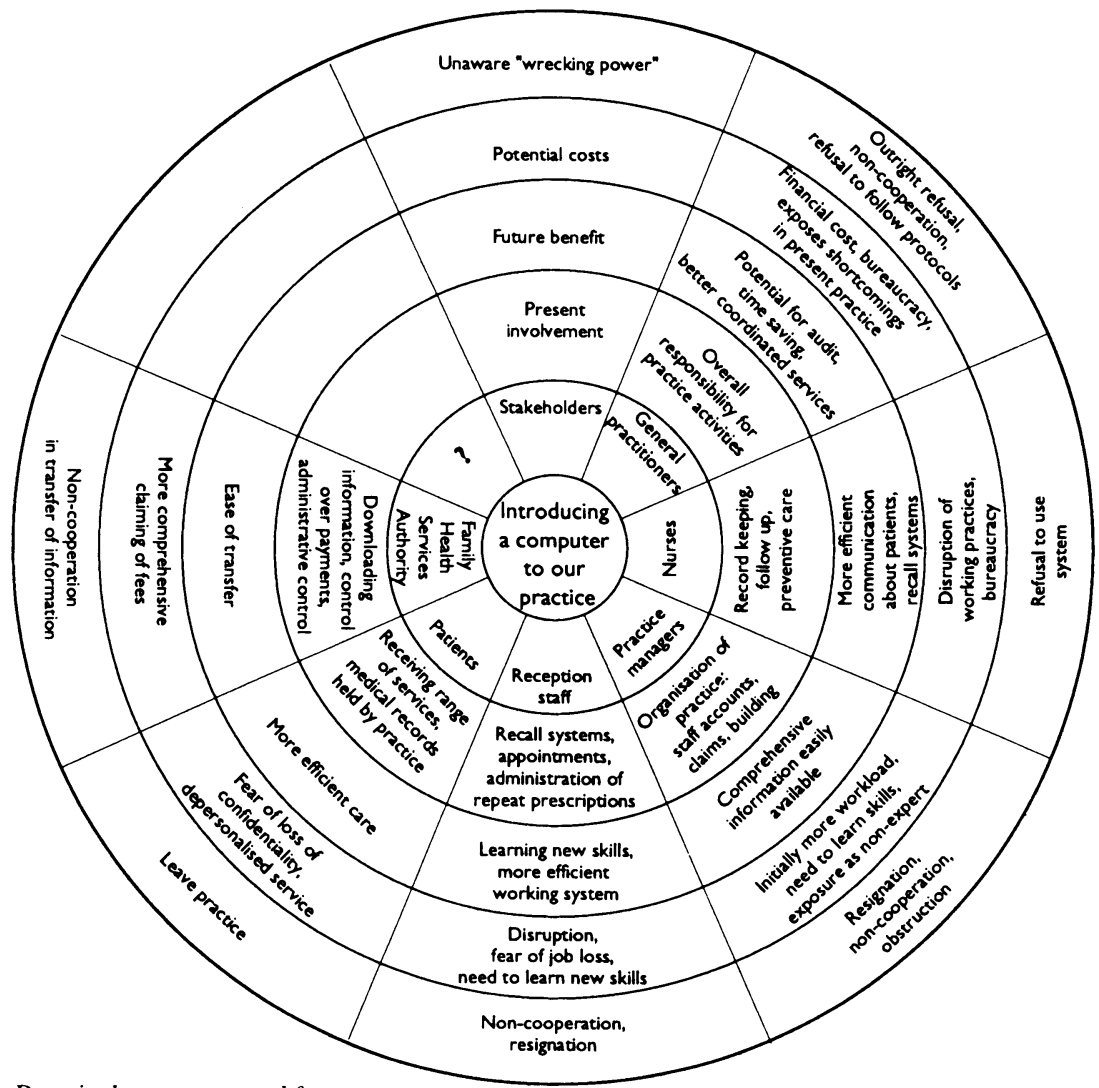

Domainal map constructed for introducing computer into a general practice structure for such discussion is to start by briefly presenting the idea and then eliciting, in turn, the benefits and costs of the innovation as perceived by the stakeholder. By asking each person to identify all others that might be affected you may also avoid overlooking important groups. The success of these discussions depends on creating collaboration rather than confrontation. Box 1 gives some techniques that have been helpful in creating such an atmosphere.

As well as generating such strategic information these discussions allow refinement of the original idea. Trusting colleagues with the idea and giving them an opportunity to contribute to its development also encourages a sense of ownership of the innovation at an early stage. Finally, in teams where joint decision making is uncommon, these discussions build the foundations for a team meeting at which perspectives can be shared and a team decision reached.

\section{Evaluating the information}

After completing the discussions with key individuals, it will be possible to evaluate the feasibility of the proposed innovation and decide whether to take the next steps. When there are several stakeholders the amount of data generated by these meetings can be unwieldy. A domainal map, a tool derived from industry, can be used to organise these data and as an aid to effective planning. ${ }^{16}$ Its usefulness lies in its visual display of each person's perceptions of the change, its exploration of the costs of the change as well as the benefits, and in making explicit each person's power to obstruct as well as promote the change. Box 2 gives instructions for constructing a domainal map and the figure shows an example of a completed map for introducing a computer into our practice.

The domainal map brings together information about the potential future benefit of the innovation to the practice and the cumulative power which the group has to support or obstruct the project. The likelihood that any individual will use this power depends on whether the future benefit is seen to outweigh the cost to that individual. This information can be used to estimate the effort which may be needed in negotiating and implementing the proposed innovation and to decide whether to take the next steps.

\section{Getting agreement}

The next step is to arrange a meeting with the whole group. The ultimate aim of the meeting is to reach public agreement about whether to proceed with the innovation. Hearing other members' views, both positive and negative, allows broader understanding of the implications of the proposed change. Achieving a public consensus, in the light of all the information which is available, leads to a more robust agreement and builds team cohesiveness. ${ }^{17}$

We have found several factors important in ensuring the success of meetings. The first is to encourage all stakeholders to attend. Non-attendance cañ be minimised by emphasising to each person how vital their contribution will be and pointing out the cost both to the person and to the group if he or she does not attend.

\section{CHAIRING THE MEETING}

The chairperson has considerable influence over the ethos of the meeting and is responsible for setting the ground rules. Our experience shows that meetings are successful when all members feel able to contribute because they know their contribution will be valued; no one member of the group, however powerful, is allowed to dominate; and there is an atmosphere of cooperation and trust. This does not mean that consensus will be easy, but rather that, when there is disagreement, members are encouraged to work together to understand it and to negotiate constructively.

Several techniques can be used to promote a collaborative and positive atmosphere. Firstly, validating each person who speaks by appreciating their contribution. Secondly, identifying members of the group who find difficulty in speaking and encouraging their contribution. Thirdly, discouraging one person or group from dominating the discussion by gently interrupting a lengthy monologue, summing up the main points, and then calling for comments from other people. Fourthly, identifying areas of disagreement and arbitrating between different factions. (Failure to 


\section{Box 3: Possible objectives for a new computer system}

Improve practice administration

Save time spent previously on manual systems

Efficient auditing of clinical care

Gain computer skills

Optimise practice income through accurate informa-

tion to meet prevention targets-for example, immunisation, cervical screening

Efficient recall for surveillance of chronic disorders

Improve administration of repeat prescriptions

Test feasibility of fundholding by forecasting prescribing costs

acknowledge and deal with disagreement leads to at least one faction being dissatisfied and compromises the faction's commitment to the outcome of the meeting. The chairperson's task is to reflect back both parties' positions and to establish any part of the proposal about which they do agree. This enables the area of disagreement to become more specific, so that negotiations can be more focused..$^{18}$ ) Lastly, defusing situations in which other group members are personally attacked - for example, by rephrasing criticism in a neutral manner to focus on the problem rather than the person.

\section{STRUCTURING THE MEETING}

An efficient structure for the meeting is essential. The aims and agenda for the meeting must be clear, and it is helpful if they can be agreed with all stakeholders in advance. The length of the meeting should be explicit and adhered to, and it may help to allocate a length of time for each agenda item.

\section{USING THE DOMAINAL MAP}

We have already described how a domainal map could be developed to summarise information collected from individuals. The same map can be used to present the group with information on which to base negotiation. We suggest that the domainal map should be large enough to be visible to the whole group. By considering the map, segment by segment, each person's perspective can be shared with the group and other group members have the opportunity to ask for clarification.

Demonstration that each of these categories applies to each person will legitimise his or her position as a stakeholder, the reason for his or her involvement, and, very importantly, his or her power. Such a public acknowledgment increases the likelihood that this power will be used responsibly.

Having established the credentials and perspectives of each person, the next step is to remind the group of the proposal in its present form. The original idea will probably have been modified considerably in the light of the information gathered and earlier discussions.

\section{MOVING TOWARDS A DECISION}

To move towards agreement an honest response to the proposal must be obtained from each member of the group. Some people will have changed their attitude to the proposal after hearing the views of other team members. A useful technique is to go round the group asking each person three simple questions: "What appeals to you about this proposal now? What concerns you about it now? and Do you need any more information in order to reach a decision?" It helps to display the questions and each person's response on a board or flip chart. Equal time should be given to each person. From this visual display the chairperson can summarise the areas of agreement and start the negotiations.
The ultimate aim is to reach an informed decision, to which all the members of the group are committed, about whether or not to implement the proposal. ${ }^{17}$ However, when agreement proves difficult it may be appropriate to reach a conditional decision-for example, "We would be happy to go ahead if we can be sure that this computer system will protect confidentiality." This makes reaching a decision easier as it leaves open the opportunity of reversing a decision in the light of new information.

Time should be left at the end of this meeting to review progress by confirming agreements reached, identifying any remaining disagreements, and deciding what steps are needed to resolve them. If complete agreement to implement the innovation has been reached it is important to clarify and agree the next steps to be taken. This will usually include setting a date for a meeting to plan the effective implementation of the innovation.

It is good practice to write down agreements reached, unresolved issues and tasks to be undertaken, and responsibility for each task. If possible these minutes should be circulated to all stakeholders after the meeting. A major decision will involve several steps, and a meeting at which some of the necessary steps are taken is still successful even if a final decision is not reached. It is as important to take the steps as to reach the decision.

\section{Implementing change}

Once agreement about the innovation has been agreed in principle, a practical design for the innovation is needed. We present this process as a series of key steps. Not all steps will be relevant to all innovations and we recommend flexibility in their use.

\section{DEFINING KEY OBJECTIVES}

The first step is to define the key objectives to meet the aims of the new system. At a team meeting this can be done by dividing into small groups, to discuss the question, "What do you think is important for this new system to achieve?" Time should be divided so that each person has equal opportunity to speak and to be listened to. The main points from these small groups can be fed back to the meeting and listed on a flip chart. They can then be discussed and agreed. Box 3 gives a possible list of such objectives for introducing a computer into a general practice.

It is helpful to gather accurate data about the current situation; this reinforces commitment to improvement, and provides motivation to change. This information also establishes a baseline against which future progress can be measured.

\section{EXPLORING THE RANGE OF OPTIONS}

Alternative ways of implementing the proposal, drawn from the initial investigations, can be shared. Further approaches can be generated by the group before agreeing on a preferred option. Box 4 gives some possible options for a new computer system.

\section{IDENTIFYING TASKS AND AGREEING RESPONSIBILITIES}

All the tasks that are necessary to set up the new system should be listed ${ }^{19}$ and agreement reached about

\section{Box 4: Options for a new computer system}

"Free" system, conditional on data collection

Reception based system with one terminal and printer Linked system with terminals in each consulting room Inclusion of financial and accounting package Coordinated by practice manager or general practitioner or specialist computer operator 
who is responsible for carrying out each one. ${ }^{20}$ It helps to have someone in a central coordinating role with responsibility for overseeing the progress of the in-• novation as a whole. It is crucial to identify the resources and skills which will be needed to fulfil individual responsibilities so that any gaps in skills or resources can be identified and strategies for filling them developed (box 5).

\section{SETTING A REALISTIC TIMETABLE}

A realistic timetable for implementation can now be planned and agreed. This helps to keep up momentum and sets a goal towards which people can work. A simple record of each step including decisions made and an "action column" to remind members of their responsibilities is useful.

\section{REVIEWING PROGRESS}

Regular review of progress is important. This offers an opportunity to address difficulties which arise in relation to the project. A useful technique at review meetings is to invite each member to respond to two questions: "What has gone well since the last meeting?" and "What has been difficult since the last meeting?" Recognising what has gone well offers both an opportunity to celebrate the successes achieved so far and a positive perspective against which the difficulties can be viewed. ${ }^{21}$ This technique also legitimises difficulties by acknowledging that they are to be expected during the process of change. It makes it safe enough for people to share their problems and have access to the constructive thinking of the group about possible solutions and next steps.

\section{DEVELOPING PRACTICE POLICIES AND GUIDELINES}

In complex groups such as the practice team efficient functioning will be enhanced by protocols for action which are agreed, understood, and implemented by all members of the group. Such guidelines promote continuity even if changes in staff occur. The design of protocols can be influenced by what has worked in other settings and by district policy or guidelines.

Protocols should include guidelines for monitoring the effectiveness of the new system based on the objectives agreed previously. In introducing a new computer the criteria for success might include both qualitative and quantitative measures such as accuracy and completeness of data, efficiency of data retrieval, time involved, and staff and patient satisfaction. There needs to be agreement about how these criteria will be assessed, who will be responsible for gathering information, and when it will be appropriate to present the results to the team.

Protocols cannot usually be finalised without trying them out, to give all members of the team the opportunity to assess their practicality, identify their strengths and weaknesses, and if necessary, to generate ideas for improvement. By a joint process of implementation and review, difficulties in organisation can be identified early and modifications can be made before problems become intractable.

\section{FORMAL REVIEW AND CELEBRATION}

A formal review meeting, possibly at the end of the first year of the innovation, is helpful. The agenda might include progress reports from team members, the results of the evaluation, celebration of the successes of the year, and agreement about further modifications if necessary.

Evaluation is most effective when used to enhance learning and future performance rather than to punish the shortcomings of the past. Celebration is particularly important, and even where an innovation has been fraught with difficulties there is always some
Box 5: Skills and resources that might be needed by a person coordinating general practice computer system

$\begin{array}{lll}\text { Resources } & \text { Skills or } & \\ \text { Time } & \text { Keybledge } & \text { Support } \\ \text { Equipment } & \text { Computer } & \text { Training courses } \\ \text { Space } & \text { literacy } & \text { other team } \\ \text { Appropriate } & \text { Understanding of } & \text { members } \\ \text { software } & \text { general practice } & \text { Other computer } \\ & \text { organisation } & \text { coordinators } \\ & \text { Communication } & \text { System helpline } \\ & \text { skills in } & \\ & \text { disseminating } & \\ & \text { information } & \end{array}$

progress. Celebration builds team cohesiveness, reasserts the ability of the team to succeed, and sets the scene for further growth and development. ${ }^{21}$

\section{Conclusion}

We have presented a well tried model for managing change in general practice. We have argued for the importance of obtaining comprehensive background information, recognising barriers to change, identifying and negotiating with all key people, achieving robust agreement to the innovation, and developing effective planning for its implementation and evaluation. Underlying all these strategies is the recognition of the strength of a team approach to change.

This work grew out of a multidisciplinary project carried out by a team funded by the Nuffield Provincial Hospitals Trust. The paper was written by Nicki Spiegal and Elizabeth Murphy and drew on insights developed with the other authors during team meetings. We thank the practice teams with whom we developed and refined the ideas presented here. Nicki Spiegal was supported by Hampshire Family Health Services Authority and by the Community Unit of Southampton and South West Hampshire Health Authority. A more detailed version of this paper is available from the authors.

1 Secretaries of State for Social Services, Wales, Northern Ireland, and Scotland. Promoting better health: the Government's programme for improving primary Promoting better health: the Government's progendon: HMSO, 1987. (Cm 249.)

2 Secretaries of State for Health, Wales, Northern Ireland, and Scotland. Working for patients. London: HMSO, 1989. (Cm 555.)

3 Health Departments of Great Britain. General practice in the National Health Service: the 1990 contract. London: Health Departments of Great Britain.

4 Royal College of General Practitioners. What sort of doctor? London: Royal College of General Practitioners, 1985

5 Royal College of General Practitioners. Quality in practice. London: Royal College of General Practitioners, 1986.

6 Plant R. Managing change and making it stick. London: Gower, 1987.

7 Petters T, Austin N. A passion for excellence. London: Collins, 1985.

8 Turrill T. Change and innovation: a challenge for the NHS. L.ondon: Institute of Health Services Management, 1986

9 Stewart R, Smith P, Blake J, Wingate P. The district administrator in the National Health Service. London: King Edward's Hospital Fund for London, 1980.

10 Stocking B. Intiative and inertia; case studies in the NHS. London: Nuffield Provincial Hospitals Trust, 1985.

11 Bain DJG, Coates R, Kinmonth AL, Murphy EA, Ross F, Spiegal NM. Optimising the care of patients with chronic disorders, project report to the Nuffield
Provincial Hospitals Trust. London: Nuffield Provincial Hospitals Trust, Provincial Hospitals Trust. London: Nuffield Provincial Hospitals Trust

12 Rogers E. Diffusion of innovations. 3rd ed. New York: Free Press, 1982.

13 Plant R, Managing change and making it stick. London: Gower, 1987:25-6.

14 Turrill T. Change and innovation: a challenge for the NHS. London: Institute of Health Services Managing, 1986:24-5.

15 Moss-Kanter R. The change masters. London: Unwin, 1983:180-205.

16 Pedlar M, Burgoyne J, Boydell T. A managers guide to self-development. 2nd ed. Maidenhead: McGraw Hill, 1986.

17 Peters T, Austin N. A passion for excellence. London: Collins, 1985:213-51. 18 Fisher R, Ury W. Getting to yes. London: Business Books, 1982.

19 Turill T Changend innoution: a chullenge for the NHS. London: Institute of Health Services Manovation: a challenge for

20 Turrill T. Change and innovation: a challenge for the NHS. London: Institute of Health Services Management, 1986:81.

21 Peters T, Austin N. A passion for excellence. London: Collins, 1985:252-62.

(Accepted 26 October 1990) 